\title{
12.
}

\section{Mémoire sur la détermination des racines primitives des nombres premiers.}

(Par Mr. Oltramare, prof. de math. supér. à l'académie des sciences de Genêve.)

Nous nous proposons dans ce mémoire de réunir plusieurs propositions à l'aide desquelles on puisse déterminer une racine primitive d'un nombre premier; car on sait que la connaissance d'une racine primitive conduit aisément à la détermination de toutes les autres racines de ce nombre premier.

Bien que l'ensemble des théorèmes que nous allons établir, ne puisse généralement donner une racine primitive de tout nombre premier proposé, cependant il en est peu dont la forme ne permette pas d'arriver à la détermination de l'une de ses racines primitives, et nous nous sommes assurés que, sur les nombres premiers plus petits que 3400 , les racines primitives de 30 seulement ne potvaient être trouvées.

\section{\$. 1.}

Théorème I. Si un nombre premier $\mu$, qu'on peut toujours supposer de la forme $2^{s} \alpha^{m} \beta^{n} \gamma^{p} \ldots \varepsilon^{q}+1(\alpha, \beta, \gamma, \ldots \varepsilon$ étant également des nombres premiers différents entr'eux et plus grands que 2) est tel, qu'en désignant par $r_{f}$ une racine de ce nombre de l'ordre t: la congruence

$$
\text { (1.) }\left(r_{t}\right)^{\alpha(\mu-1)\left\{\frac{1}{\beta}+\frac{1}{\gamma}+\cdots+\frac{1}{\varepsilon}\right\}}-1 \equiv 0(\bmod \mu)
$$

n'est point satisfaite.

En posant:

$$
\boldsymbol{R}_{k} \equiv \cos \frac{2 k \omega}{\alpha}+\sin \frac{2 k \omega}{\alpha} \sqrt{ }-1 \quad(\bmod . \mu)
$$

( $k$ étant un nombre entier compris entre 0 et $\alpha$ ): la valeur de l'expression

$$
\text { (2.) } x \equiv\left(\boldsymbol{r}_{f}\right)^{\alpha}{ }^{\alpha^{m-1}} \boldsymbol{R}_{k} \quad(\bmod . \mu)
$$

sera une racine du nombre premier $\mu$ d'un ordre marqué par le plus grand commun diviseur entre $t$ ef $2^{s}$. 
Pour reconnaître l'exactitude de ce théorème, nous remarquerons d'abord que la valeur

$$
x \equiv\left(\boldsymbol{r}_{t}\right)^{\alpha} \sqrt{\boldsymbol{\alpha}^{m-1}} \boldsymbol{R}_{k} \quad(\bmod . \mu)
$$

ne peut satisfaire à aucune des congruences qui donnent des racines du nombre premier $\mu$ d'un ordre dont l'indice est un multiple de $\alpha$; par la raison que l'expression

$$
\stackrel{a}{\sqrt{ } \boldsymbol{x}} \equiv \boldsymbol{r}_{t} \cdot \boldsymbol{\alpha}^{m} \boldsymbol{R}_{k} \quad(\bmod . \mu)
$$

est une quantité irrationnelle pour tout nombre premier $\mu$ qui n'est pas de la forme $\alpha^{m-1} h+1$.

En second lieu, si nous supposons qu'on ait formé les congruences qui donnent les racines du nombre premier $\mu$ des ordres non multiples de $\alpha$, et qu'on $\mathrm{y}$ ait substitué pour $x$ la valeur $\left(\boldsymbol{r}_{t}\right)^{\alpha} \sqrt{\alpha^{m-1}} \boldsymbol{R}_{k}$, en admettant que cette valeur puisse satisfaire à l'une d'elles, on arrivera nécessairement au résultat suivant:

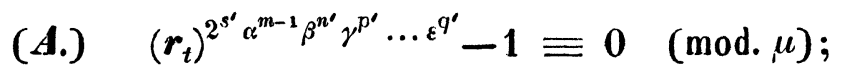

relation dans laquelle

$s^{\prime}$ sera plus petit ou tout au plus égal à $s$,

$n^{\prime}$ sera plus petit ou tout au plus égal à $n$,

$q^{\prime}$ sera plus petit ou tout au plus égal à $q$.

Mais cette dernière congruence sera cependant telle que l'on ne saurait avoir simultanément:

$$
n^{\prime}=n, \quad p^{\prime}=p, \ldots \ldots q^{\prime}=q,
$$

si l'on admet de laisser de côté les congruences

(B.)

$$
\left.\left\{\begin{array}{l}
\boldsymbol{X}_{\left(2^{s}\right)}=\frac{x^{\alpha^{m} \beta^{n} \gamma^{p} \ldots \varepsilon^{q}-1}}{\boldsymbol{X}} \equiv 0 \\
\boldsymbol{X}_{\left(2^{s-1}\right)}=\frac{x^{2 \alpha^{m} \beta^{n} \gamma^{p} \ldots \varepsilon^{q}-1}}{\boldsymbol{X}^{\prime}} \equiv 0 \\
\ldots \ldots \ldots \ldots \ldots \ldots \ldots \ldots \ldots \varepsilon^{q}-1 \\
\boldsymbol{X}_{(2)}=\frac{x^{2^{s-1} \alpha^{m} \beta^{n} \gamma^{p} \ldots \ldots}}{\boldsymbol{X}^{(s-1)}} \equiv 0 \\
\boldsymbol{X}_{(1)}=\frac{x^{2^{s-1} \alpha^{m} \beta^{n} \gamma^{p} \ldots \varepsilon^{q}+1}}{\boldsymbol{X}^{(s)}} \equiv 0
\end{array}\right\} \text { (mod. } \mu\right)
$$


qui déterminent les racines du nombre premier $\mu$ des ordres $2^{s}, 2^{s-1}, \ldots 2$ et primitives.

Il résulte de là que si aucune des congruences

$$
\text { (C.) }\left\{\begin{array}{l}
\left(\boldsymbol{r}_{t}\right)^{\alpha(\mu-1) \frac{1}{\beta}}-1 \equiv 0 \quad(\bmod . \mu), \\
\left(\boldsymbol{r}_{t}\right)^{\alpha(\mu-1) \frac{1}{\gamma}}-1 \equiv 0 \quad(\bmod . \mu), \\
\ldots \ldots \ldots \ldots \\
\left(\boldsymbol{r}_{t}\right)^{\alpha(\mu-1) \frac{1}{\varepsilon}}-1 \equiv 0 \quad(\bmod . \mu),
\end{array}\right.
$$

n'est satisfaite, il faudra en conclure, à fortiori, que la congruence (A.) ne peut ètre satisfaite, et par conséquent que l'expression

$$
\boldsymbol{x} \equiv\left(\boldsymbol{r}_{t}\right)^{\alpha} \sqrt{\alpha^{m-1}} \boldsymbol{R}_{k} \quad(\bmod . \mu)
$$

est nécessairement une racine d'un ordre marqué par l'une des congruences ( $\boldsymbol{B}$.).

Si l'on remarque que l'ensemble des congruences $(C$.) peut être mis sous la forme de la relation unique

$$
\left(r_{t}\right)^{\alpha(\mu-1)\left(\frac{1}{\beta}+\frac{1}{\gamma}+\cdots+\frac{1}{\varepsilon}\right)}-1 \equiv 0 \quad(\bmod . \mu),
$$

on en conclura que, si cette dernière congruence n'est pas satisfaite, l'expression $\left(\boldsymbol{r}_{t}\right)^{\alpha} \sqrt{\alpha^{m-1}} \boldsymbol{R}_{k}$ est une racine du nombre premier $\mu$ de l'un des ordres $2^{s}, 2^{s-1}, \ldots 2$, ou primitive.

En mettant cette expression dans les congruences $(\boldsymbol{B}$.) on obtient:

$$
\begin{aligned}
& \left(\boldsymbol{r}_{t}\right)^{\alpha^{m+1} \beta^{n} \gamma^{p} \ldots \varepsilon^{q}}-1 \equiv 0 \quad(\bmod . \mu), \\
& \left(\boldsymbol{r}_{t}\right)^{2 \alpha^{m+1} \beta^{n} \gamma^{p} \ldots \varepsilon^{\varphi}}-1 \equiv 0 \quad(\bmod . \mu) \text {, }
\end{aligned}
$$

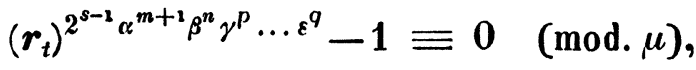

$$
\begin{aligned}
& \left(\boldsymbol{r}_{t}\right)^{2^{s-1} \alpha^{m+1} \beta^{n} \gamma^{p}}: \cdots \varepsilon^{q}+1 \equiv 0 \quad(\bmod . \mu) ;
\end{aligned}
$$

et on peut conclure de là que :

$1^{\circ}$. Si $r_{t}$ est une racine impaire de $\mu$, ou $t$ un nombre impair, c'est la dernière de ces congruences qui est satisfaite; par suite l'expression $\left(\boldsymbol{r}_{t}\right)^{\alpha}{\sqrt{\boldsymbol{R}_{k}}}^{m-1}$ est une racine primitive du nombre premier $\mu$. 
$2^{\circ}$ Si $r_{t}$ est une racine de $\mu$ d'un ordre seulement une fois divisible par 2 , c'est l'avant-dernière de ces congruences qui est satisfaite, et par suite l'expression $\left(\boldsymbol{r}_{t}\right)^{\alpha}{ }^{\alpha^{m-1}} \boldsymbol{R}_{k}$ est une racine seconde de $\mu$.

$3^{\circ}$. Si $r_{t}$ est une racine de $\mu$ d'un ordre doublement pair, l'expression $\left(\boldsymbol{r}_{t}\right)^{\alpha} \sqrt{\boldsymbol{a}^{m-1}} \boldsymbol{R}_{k}$ est une racine de l'ordre $2^{2}$.

$4^{\circ}$. Si etc.

En d'autres termes, l'expression $\left(\boldsymbol{r}_{t}\right)^{\alpha} \gamma^{\alpha^{m-1}} \boldsymbol{R}_{k}(\bmod . \mu)$ est une racine d'un ordre marqué par le plus grand commun diviseur entre $t$ et $2^{s}$.

On peut substituer à la congruence (1.) une congruence beaucoup plus simple, en remarquant:

$1^{\circ}$. Que par le théorème de Fermat on a:

$$
\left(r_{t}\right)^{\mu-1}-1 \equiv 0 \quad(\bmod . \mu) \text {, }
$$

$2^{\circ}$. Que les valeurs de $r_{t}$ qui satisfont à la congruence (1.) et à celle-ci, doivent également satisfaire à la congruence

$$
\left(r_{t}\right)^{D}-1 \equiv 0 \quad(\bmod . \mu)
$$

$D$ représentant le plus grand commun diviseur entre $\alpha(\mu-1)\left\{\frac{1}{\beta}+\frac{1}{\gamma}+\cdots+\frac{1}{\varepsilon}\right\}$ et $\mu-1$ qui a pour valeur:

$$
D=2^{s} \alpha^{m} \beta^{n-1} \gamma^{p-1} \ldots \varepsilon^{q-1} .
$$

On pourra done au lieu de la congruence (1.) employer la suivante:

$$
\left(\boldsymbol{r}_{t}\right)^{2^{s} \alpha^{m} \beta^{n-1} \gamma^{p-1} \ldots \varepsilon^{q-1}}-1 \equiv 0 \quad(\bmod . \mu) \text {. }
$$

\$. 2 .

Les valeurs de

$$
\boldsymbol{R}_{k} \equiv \cos \frac{2 k \omega}{\alpha}+\sin \frac{2 k \omega}{\alpha} \sqrt{ }-1 \quad(\bmod . \mu)
$$

considérées dans le paragraphe précédent, n'étant autre chose que les racines de la congruence

$$
x^{\alpha}-1 \equiv 0 \quad(\bmod . \mu)
$$

différentes de l'unité, î est évident que si al'on désigne par $g$ un nombre tel que la congruence

$$
g^{\frac{\mu-1}{\alpha}}-1 \equiv 0 \quad(\bmod . \mu)
$$

ne soit point satisfaite, en posant

$$
\boldsymbol{x} \equiv \boldsymbol{R}_{k} \equiv g^{\frac{\mu-1}{\alpha}},(\bmod . \mu)
$$


on aura une racine de la congruence proposée, différente de l'unité. Cette valeur de $\boldsymbol{R}_{k}$, mise dans la congruence (2.) du parag. précédent, nous permet d'établir la proposition suivante:

Théorème II. Si un nombre premier $\mu$ qu'on peut supposer de la forme $2^{s} \alpha^{m} \beta^{n} \gamma^{p} \ldots \varepsilon^{q}+1$, est tel que:

$1^{\circ}$. En désignant par $r_{t}$ une racine impaire de $\mu$, la congruence

$$
\left(\boldsymbol{r}_{t}\right)^{2^{s} \alpha^{m} \beta^{n-1} \gamma^{p-1} \ldots \varepsilon^{q-1}-1 \equiv 0 \quad(\bmod . \mu)}
$$

n'est point satisfaite,

$2^{\circ}$. En designant par g un nombre tel que la congruence

$$
g^{\frac{\mu-1}{\alpha}}-1 \equiv 0 \quad(\bmod . \mu)
$$

n'est point satisfaite, la valeur de l'expression

$$
x \equiv\left(\boldsymbol{r}_{t}\right)^{a} g^{\frac{\mu-1}{\alpha^{m}}}(\bmod . \mu)
$$

sera une racine primitive du nombre premier $\mu$.

Si l'on suppose $\mu=2 \alpha \beta+1, \alpha$ et $\beta$ étant différents de $3, \mu$ sera de la forme $6 n-1$, et -3 sera une racine impaire de $\mu$. On aura donc, en faisant $g=-3$, la proposition suivante:

Théorème a. Si $\mu$ est un nombre premier de la forme $2 \alpha \beta+1$ ( $\alpha$ et $\beta$ étant différents de 3 ):

$1^{\circ}$. La valeur de l'expression

$$
-3^{a} 2^{2 \beta} \quad \text { (mod. } \mu \text { ) }
$$

sera racine primitive de $\mu$, si aucune des deux congruences

n'est satisfaite.

$$
\begin{array}{ll}
3^{2 \alpha}-1 \equiv 0 & (\bmod . \mu) \\
2^{2 \beta}-1 \equiv 0 & \text { et }
\end{array}
$$

$2^{\circ}$. La valeur de l'expression

$$
-3^{\beta} 2^{2 \alpha} \quad(\bmod . \mu)
$$

sera racine primitive de $\mu$, si aucune des deux congruences

n'est satisfaite.

$$
\begin{array}{ll}
3^{2 \beta}-1 \equiv 0 & (\bmod \mu) \\
2^{2 \alpha}-1 \equiv 0 & \text { et }
\end{array}
$$

On peut déduire comme cas particuliers: 
Théorème $a^{\prime}$. Si $\mu$ est un nombre premier de la forme $2.11 . \beta+1$ ( $\beta$ étant différent de 3 ), la valeur de l'expression

$$
-3^{11} \cdot 2^{2 \beta} \quad(\bmod . \mu)
$$

sera une racine primitive de $\mu$, si la congruence

$$
2^{2 \beta}-1 \equiv 0 \quad(\bmod . \mu)
$$

n'est pas satisfaite.

Théorème $a^{\prime \prime}$. Si $\mu$ est un nombre premier de la forme $2.11 . \beta+1$ ( $\beta$ étant différent de 3 et 31 ), la valeur de l'expression

$$
-3^{\beta} \cdot 2^{22} \quad(\bmod . \mu)
$$

sera une racine primitive de $\mu$, si la congruence

n'est pas satisfaite.

$$
3^{2 \beta}-1 \equiv 0 \quad(\bmod . \mu)
$$

Théorème $b^{\prime}$. Si $\mu$ est un nombre premier de la forme $2.13 . \beta+1$ ( $\beta$ étant différent de 3 ), la valeur de l'expression

$$
-3^{13} \cdot 2^{2 \beta} \quad(\bmod . \mu)
$$

sera une racine primitive de $\mu$, si la congruence

$$
2^{2 \beta}-1 \equiv 0 \quad(\bmod . \mu)
$$

n'est pas satisfaite.

Théorème b". Si $\mu$ ést un nombre premier de la forme $2.13 \beta+1$ ( $\beta$ étant différent de 3 ), la valeur de l'expression

$$
-3^{\beta} .2^{26}(\bmod , \mu)
$$

sera une racine primitive de $\mu$, si la congruence

n'est pas satisfaite.

$$
3^{2 \beta}-1 \equiv 0 \quad(\bmod . \mu)
$$

\$. 3.

$\mathrm{Si}$, dans le théorème (II.), on admet qu'il puisse exister une valeur de $g$ égale à $r_{t}$, on obtient le théorème suivant:

Théorème III. Si $r_{t}$ est une racine impaire du nombre premier $\mu$ de la forme $2^{s} \alpha^{m} \beta^{n} \gamma^{p} \ldots \varepsilon^{q}+1$, telle que les congruences

$$
\begin{aligned}
&\left(\boldsymbol{r}_{t}\right)^{\frac{\mu-1}{\alpha}}-1 \equiv 0 \quad(\bmod \mu) \text { et } \\
&\left(\boldsymbol{r}_{t}\right)^{s^{s} \alpha^{m} \beta^{n-1} \gamma^{p-1} \ldots \varepsilon^{q-1}}-1 \equiv 0 \quad(\bmod \mu \mu)
\end{aligned}
$$

ne soient point satisfaites; celle vuleur de $r_{t}$ sera une racine primilive de $\mu$. 
En remarquant que, si

$$
x \equiv\left(\boldsymbol{r}_{t}\right)^{\alpha+\frac{\mu-1}{\alpha^{m}}} \quad(\bmod . \mu)
$$

est une racine primitive de $\mu, r_{t}$ doit également être une racine primitive de ce même nombre premier. 0 n en peut déduire de ce théorème comme cas particulier:

Théorème a. Le nombre -3 est racine primitive de tout nombre premier $\mu=2^{s} \alpha \beta+1$ ( $\alpha$ et $\beta$ étant différents de 3), si aucune des congruences

n'est sutisfuite.

$$
\begin{aligned}
& 3^{2^{s} \alpha}-1 \equiv 0(\bmod . \mu) \text { et } \\
& 3^{2^{s} \beta}-1 \equiv 0(\bmod . \mu)
\end{aligned}
$$

S. 4.

Supposant le nombre premier $\mu$ de la forme $2^{s} \alpha^{m}+1$, il n'existera aucune racine du nombre premier $\mu$ d'un ordre non multiple de $\alpha$, autre que les racines des ordres $2^{s}, 2^{s-1}, \ldots 2$ et primitives, et l'on pourra substituer au théorème (I.) le suivant:

Théorème IV. Si $\mu$ est un nombre premier de la forme $2^{s} \alpha^{m}+1$ ( $\alpha$ étant également un nombre premier), en désignant par $\boldsymbol{r}_{t}$ une racine de ce nombre de l'ordre $t$, et en posant:

$$
\boldsymbol{R}_{k} \equiv \cos \frac{2 k \omega}{\alpha}+\sin \frac{2 k \omega}{a} \sqrt{ }-1 \quad(\bmod . \mu)
$$

la valeur de l'expression

$$
\left(\boldsymbol{r}_{t}\right)^{\alpha} \sqrt{\alpha^{m-1}} \boldsymbol{R}_{k} \quad(\bmod . \mu)
$$

sera une racine du nombre premier $\mu$ d'un ordre marqué par le plus grand commun diviseur entre $t$ et $2^{s}$.

En combinant ce théorème avec le théorème (III.) du parag. précédent, on obtient:

Théor ème $V$. Si $r_{t}$ est une racine impaire du nombre premier $\mu$ de la forme $2^{s} \alpha^{m}+1$, telle que la congruence

$$
\left(r_{t}\right)^{\frac{\mu-1}{a}}-1 \equiv 0 \quad(\bmod . \mu)
$$

n'est pas satisfnite, cette valeur de $r_{t}$ sera une racine primitive du nombre premier $\mu$.

Crelle's Journal f. d. M. Bd. XLIX. Heft 2. 
S. 5.

Les valeurs de $\boldsymbol{R}_{k}$, ou les racines de la congruence

$$
x^{\alpha}-1 \equiv 0 \quad(\bmod . \mu) \text {, }
$$

différentes de l'unité, peuvent être trouvées pour certaines valeurs de $\alpha$ par des formules faciles à calculer.

$1^{\circ}$. Soit $\alpha=3$, on a :

$$
\boldsymbol{R}_{k} \equiv-\frac{1}{2} \pm \frac{1}{2} \gamma-3 \quad(\bmod . \mu) \text {. }
$$

Pour déterminer la valeur de l'expression $\gamma-3(\bmod \mu)$ qui, par la forme du nombre premier $\mu$, est rationnelle; il suffit de mettre le nombre premier $\mu$ sous la forme $q^{2}+3 p^{2}$ et de poser

$$
\sqrt{ }-3 \equiv 4 y_{1}-3 p x_{1} \quad(\bmod . \mu),
$$

$x_{1}$ et $y_{1}$ étant une solution particulière de l'équation indéterminée

$$
q x+p y=1 .
$$

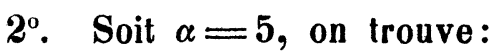

$$
\boldsymbol{R}_{k} \equiv-\frac{1}{4}+\frac{1}{4} \sqrt{5}+\frac{1}{4} l^{\prime}(-10-2 \sqrt{5}) \quad(\bmod . \mu) .
$$

Pour déterminer la valeur de l'expression $/ 5(\bmod . \mu)$ qui, par la forme du nombre premier $\mu$, est rationnelle, il suffit de mettre le nombre premier $\mu$ sous la forme $q^{2}-5 p^{2}$ et de poser

$$
\sqrt{5} \equiv q y_{1}+5 p x_{1} \quad(\bmod . \mu),
$$

$x_{1}$ et $y_{1}$ étant une solution particulière de l'équation indéterminée

$$
q x+p y=1 \text {. }
$$

On aura ainsi:

$$
\boldsymbol{R}_{k} \equiv \frac{1}{4}\left\{q y_{1}+5 p x_{1}-1+\sqrt{ }\left(-10-2\left(q y_{1}+5 p x_{1}\right)\right)\right\} \quad(\bmod . \mu) .
$$

La valeur du radical

$$
\sqrt{ }\left(-10-2\left(q y_{1}+5 p x_{1}\right)\right) \equiv \sqrt{ } K(\bmod . \mu)
$$

s'obtiendra de même en posant

et en posant:

$$
\mu=q^{\prime 2}-k p^{\prime 2}
$$

$$
\sqrt{ }\left(-10-2\left(q y_{1}+5 p x_{1}\right)\right) \equiv \sqrt{K} \equiv q^{\prime} y_{1}^{\prime}+l k p^{\prime} x_{1}^{\prime} \quad(\bmod . \mu),
$$

$y_{1}^{\prime}$ et $x_{1}^{\prime}$ étant une solution particulière de l'équation indéterminée

$$
g^{\prime} x^{\prime}+p^{\prime} y^{\prime}=1
$$


12. Oltramare, sur les racines primitives.

$3^{\circ}$. Soit $\alpha=7$ on obtient:

$$
\boldsymbol{R}_{k} \equiv \frac{1}{2} z+\frac{1}{2} V\left(z^{2}-4\right) \quad(\bmod . \mu),
$$

$z$ étant donné par la relation

$$
z \equiv \frac{1}{3}(v-1) \quad(\bmod . \mu)
$$

$v$ désignant une racine de la congruence du troisième degré

$$
v^{3}-21 v-7 \equiv 0 \quad(\bmod . \mu) \text {. }
$$

S. 6 .

Théorème VI. Le nombre 3 est racine primitive de tout nombre premier $\mu$ de la forme $2^{m}+1$.

Remarquons d'abord que le nombre $2^{m}+1$ ne peut être un nombre premier qu'autant que $m$ est un nombre pair; car en supposant $m=2 k+1$, on a l'expression $2^{2 k+1}+1$, qui est divisible par 3 . Si donc on pose $\mu=2^{2 k}+1$, il est facile de voir que $\mu$ est également de la forme $12 h+5$.

Or, comme le nombre 3 est racine impaire de tout nombre premier $\mu$ de la forme $12 h+5$; que d'ailleurs tonte racine impaire de $\mu$ est par la forme même de ce nombre une racine primitive: 3 est racine primitive de $\mu$.

\$. 7.

Théorème VII. Le nombre 2 est racine primitive de tout nombre premier $\mu$ de la forme $2 \alpha+1$ ( $\alpha$ étant également un nombre premier de la forme $4 n+1$ ).

L'expression $-\frac{1}{2}(\bmod . \mu)$, ou le nombre $\alpha$, est racine primitive de tout nombre premier $\mu$ de la forme $2 \alpha+1$ ( $\alpha$ étant également un nombre premier de la forme $4 n+3$ ).

En effet, tout nombre premier $\mu$ de la forme $2 \alpha+1$ ne peut avoir que des racines dont l'ordre est marqué par les nombres $1,2, \alpha$ et $2 \alpha$; mais 1 est la seule racine de l'ordre $2 \alpha$, et $\mu-1$ la seule racine de l'ordre $\alpha$; donc tous les nombres compris entre 1 et $\mu-1$ sont, ou des racines du second ordre, ou des racines primitives. Il en résulte que toute racine impaire, comprise entre ces nombres, est une racine primitive.

Si $\alpha$ est de la forme $4 n+1, \mu$ sera de la forme $8 h+3$. Mais comme 2 est racine impaire de tout nombre premier de cette dernière forme, 2 sera racine primitive de $\mu$.

Si $\alpha$ est de la forme $4 n+3, \mu$ sera de la forme $8 h+7$; mais comme 2 est racine paire de tout nombre premier de cette forme, 2 sera une racine 
seconde de $\mu$; on aura donc:

$$
2^{\frac{1}{(\mu-1)}}-1 \equiv 0 \quad(\bmod . \mu) \text {. }
$$

Cette congruence, combinée avec la congruence identique

donne

$$
2 \alpha+1 \equiv 0 \quad(\bmod . \mu) \text {, }
$$

$$
\alpha^{\frac{1}{3}(\mu-1)}+1 \equiv 0, \quad\left(-\frac{1}{2}\right)^{\frac{1}{((\mu-1)}}+1 \equiv 0 \quad(\bmod . \mu) ;
$$

$\alpha$, ou $-\frac{1}{2}(\bmod , \mu)$, étant racine impaire de $\mu$, sera une racine primitive.

\$. 8.

Théorème VIII. Le nombre 2 est racine primitive de lout nombre premier $\mu$ de la forme $2^{2} \alpha+1$ ( $\alpha$ étant également un nombre premier quelconque).

Par la forme du nombre premier $\mu=2^{2} \alpha+1$, on a les congruences

(1.) $X_{(\alpha)}=x^{2}+1 \equiv 0 \quad(\bmod . \mu)$,

(2.) $\quad X_{(1)}=\frac{x^{2 \alpha}+1}{x^{2}+1} \equiv 0 \quad$ (mod. $\left.\mu\right)$ :

la première pour déterminer les racines du nombre premier $\mu$ de l'ordre $\alpha$, la seconde pour déterminer les racines primitives.

Cela posé, si nous remarquons que le nombre 2 est une racine impaire de $\mu$, et que ce nombre ne satisfait pas à la congruence (1.), comme le nombre premier $\mu$ n'a pas d'autres racines impaires que des racines de l'ordre $\alpha$ et des racines primitives, il faudra admettre que 2 est une racine primitive de $\mu$.

Le nombre $\alpha$ ne pouvant avoir que les deux formes $4 n+1$ et $4 n+3$, il en résulte que $\mu$ est nécessairement de la forme $8 h+5$, et par suite le nombre 2 est racine impaire de $\mu$.

Faisant $x=2$ dans la congruence (1.), on obtient:

$$
5 \equiv 0 \quad(\bmod . \mu)
$$

qui ne peut être satisfaite que par $\mu=5$; et comme dans ce cas encore le nombre 2 est racine primitive de $\mu$, on en conclura le théorème que nous venons d'énoncer.

\$. 9.

Théorème IX. Si $\mu$ est un nombre premier de la forme $2^{m} .3+1$, il pourra également être mis sous la forme $\dot{q}^{2}+3 p^{2}$, et en désignant par $x_{1}$ et $y_{1}$ une solution particulière de l'équation indéterminée

$$
q x+p y=1
$$


la valeur de l'expression:

$$
5^{3}\left(-\frac{1}{2}+\frac{1}{2}\left(q y_{1}-3 p x_{1}\right)\right) \quad(\bmod . \mu)
$$

représentera une racine primitive du nombre premier $\mu$.

Remarquons d'abord que le nombre 5 est une racine impaire du nombre premier $\mu=2^{m} .3+1$; car, tout nombre premier de cette forme est nécessairement aussi de la forme $10 h \pm 3$. Faisons observer en outre que l'expression

$$
5^{3}\left(-\frac{1}{2}+\frac{1}{2} \sqrt{-3}\right) \quad(\bmod . \mu)
$$

est une racine primitive de $\mu$.

En effet, cette expression est racine impaire de $\mu$; car substituée dans la congruence

elle donne

$$
x^{\frac{1}{(\mu-1)}}+1 \equiv 0 \quad(\bmod . \mu)
$$

$$
5^{\frac{1}{((\mu-1)}}+1 \equiv 0 \quad(\bmod . \mu)
$$

et comme 5 est racine impaire de $\mu$, celte dernière congruence est satisfaite.

En second lieu, en remarquant que les racines impaires de $\mu$ ne peuvent être (par la forme même de ce nombre premier), que des racines du troisième ordre, oll des racines primitives, et que l'expression

$$
5^{3}\left(-\frac{1}{2}+\frac{1}{2} \sqrt{ }-3\right) \quad(\bmod . \mu)
$$

ne peut représenter une racine du troisième ordre, parceque la quantité

$$
\gamma^{3}\left(5^{3}\left(-\frac{1}{2}+\frac{1}{2} \sqrt{ }-3\right)\right)=5 i^{3}\left(-\frac{1}{2}+\frac{1}{2} \sqrt{ }-3\right)
$$

est irrationnelle pour tout nombre premier $\mu$ qui n'est pas de la forme $3^{2} h+1$, il faut en conclure que

$$
\text { (1.) } \quad 5^{3}\left(-\frac{1}{2}+\frac{1}{2} \gamma-3\right) \quad(\bmod . \mu)
$$

est une racine primitive de $\mu$.

or $\sqrt{ }-3$, par la forme du nombre premier $\mu$, est une quantité rationnelle dont la valeur est $q y_{1}-3 p x, p$ et $q$ étant deux nombres satisfaisant à l'égalité $\mu=q^{2}+3 p^{2}$, et $x_{1}$ et $y_{1}$ une solution particulière de l'équation indéterminée du premier degré

$$
q x+p y=1
$$

en mettant cette valeur de $\sqrt{ }-3$ dans l'expression (1.), on obtient la valeur

$$
5^{3}\left(-\frac{1}{2}+\frac{1}{2}\left(q y_{1}-3 p x_{1}\right)\right) \quad(\bmod . \mu)
$$

comme racine primitive du nombre premier $\mu$. 
S. 10.

Théorème $X$. Si le nombre premier $\mu$ de la forme $2^{m} \alpha+1$ (m étant $>1$ et $\alpha$ un nombre premier) est tel, que la congruence

$$
\text { (1.) } \quad 3^{2^{m-1}}+1 \equiv 0 \quad(\bmod . \mu)
$$

n'est point satisfaite: le nombre 3 sera racine primitive de ce nombre premier.

Par la forme du nombre premier $\mu$, on a les congruences:

$$
\begin{aligned}
& \text { (a.) } x^{2^{m-1}}+1 \equiv 0 \quad(\bmod \mu), \\
& \text { (b.) } \frac{x^{2^{m-1} \alpha}+1}{x^{2^{m-1}}+1} \equiv 0 \quad(\bmod . \mu),
\end{aligned}
$$

la première pour déterminer les racines de l'ordre $\alpha$, la seconde pour déterminer les racines primitives de $\mu$.

Cela posé, si nous remarquons que 3 est racine impaire de $\mu$ et que ce nombre ne satisfait pas à la congruence (a.); et comme le nombre premier $\mu$ n'a pas d'autres racines impaires que des racines de l'ordre $\alpha$ et des racines primitives, il faudra admettre que 3 est racine primitive de $\mu$.

Remarquons d'abord que si $m$ est' un nombre impair, $\alpha$ doit être de la forme $3 t+2$, et que si $m$ est un nombre pair, il doit être de la forme $3 t+1$.

Soient, en effet, $m=2 p+1$ et $\alpha=3 t+1$, la valeur de $\mu$ pourra s'écrire :

$$
\mu=3.2^{2 p+1} t+2^{2 p+1}+1=3 \boldsymbol{H}
$$

ce qui est impossible, puisque $\mu$ est, par hypothèse, un nombre premier.

Soient, en second lieu, $m=2 p$ et $\alpha=3 t+2$, la valeur de $\mu$ pourra s'écrire :

$$
\mu=3.2^{2 p} t+2^{2 p+1}+1=3 G,
$$

ce qui est inadmissible.

Le nombre 3 est racine impaire de tout nombre premier $\mu$ de la forme $12 h+5$. Si donc nous posons

$$
2^{m} \alpha+1=12 h+5
$$

et si, quelle que soit la valeur du nombre premier $\alpha$, on reconnait que l'on peut satisfaire à cette égalité, le nombre 3 sera racine impaire de $\mu$.

Considérons deux cas: selon que $m$ est pair, ou impair. Soit $m$ pair, $\alpha$ est de la forme $3 t+1$, et l'égalité précédente devient:

$$
h=2^{m-2} t+\frac{2^{m-2}-1}{3},
$$


qui sera satisfaite, si $m$ est égal ou plus grand que 2 ; car alors $\frac{2^{m-2}-1}{3}$ est un nombre entier si $m$ est pair. Soit $m$ impair, $\alpha$ est de la forme $3 t+2$ et l'égalité précédente devient:

$$
h=2^{m-2} \cdot t+\frac{2^{m-1}-1}{3}:
$$

égalité qui est satisfaite si m est un nombre impair plus grand que 2.

Faisons maintenant dans la congruence $(a) x=$.3 , nous aurons:

$$
3^{2^{m-1}}+1 \equiv 0 \quad(\bmod . \mu) \text {. }
$$

Il résulte de là que si cette dernière congruence n'est point satisfaite, le nombre 3 est racine primitive de $\mu$.

Théorème a. Le nombre 3 est racine primitive de tout nombre premier $\mu$ de la forme $2^{2} \alpha+1$ ( $\alpha$ étant un nombre premier plus grand que 3).

Théorème b. Le nombre 3 est racine prinitive de tout nombre premier $\mu$ de la forme $2^{3} \alpha+1$ ( $\alpha$ étant un nombre premier plus grand que 5).

Théorème c. Le nombre 3 est racine primitive de tout nombre premier $\mu$ de la forme $2^{4} \alpha+1$ ( $\alpha$ étant un nombre premier quelconque).

Théorème d. Le nombre 3 est racine primitive de tout nombre premier $\mu$ de la forme $2^{5} \alpha \nmid 1$ ( $\alpha$ étant un nombre premier plus grand que 3).

Théor ème e. Le nombre 3 est racine primitive de tout nombre premier $\mu$ de la forme $2^{6} \alpha+1$ ( $\alpha$ étant un nombre premier $>3$ ).

Théorème $f$. Le nombre 3 est racine primitive de tout nombre premier $\mu$ de la forme $2^{7} \alpha+1$ ( $\alpha$ étant un nombre premier).

Théorème g. Le nombre 3 est racine primitive de tout nombre premier $\mu$ de la forme $2^{8} \alpha+1$ ( $\alpha$ étant un nombre premier).

Théorème h. Le nombre 3 est racine primitive de tout nombre premier $\mu$ de la forme $2^{9} \alpha+1$ ( $\alpha$ étant un nombre premier).

Etc. etc.

Il suffit pour démontrer ces divers propositions 'de s'assurer que la congruence (1.) n'est point satisfaite.

๑. 11.

Théorème XI. Si le nombre $r$ est une racine impaire d'un nombre premier $\mu$ de la forme $2^{2} \alpha^{2}+1$, tel que la congruence

$$
r^{2 \alpha}+1 \equiv 0 \quad(\bmod . \mu)
$$


n'est point satisfaite: cette valeur $r$, supposée différente de $\pm 2 \alpha$, sera une racine primitive de $\mu$.

En effet, le nombre premier $\mu$ n'admettant comme racines impaires que des racines des ordres $\alpha^{2}$, $\alpha$, et primitives, si l'on remarque que la racine impaire $r$ ne satisfait à aucune des congruences

$$
\begin{array}{ll}
\text { (1.) } \quad X_{\left(\alpha^{2}\right)}=x^{2}+1 \equiv 0 \quad(\bmod . \mu), \\
\text { (2.) } \quad X_{(\alpha)}=\frac{x^{2 \alpha}+1}{x^{2}+1} \equiv 0 \quad(\bmod . \mu),
\end{array}
$$

on en conclura que $r$ est racine primitive de $\mu$.

Or, la première de ces congruences donne

$$
x \equiv \pm 2 \alpha \quad(\bmod . \mu)
$$

par consequent la valeur $r$, différente dé $\pm 2 \alpha$, ne peut y satisfaire. De plus, en supposant $x=r$ dans la congruence (2.), on a:

$$
r^{2 \alpha}+1 \equiv 0 \quad(\bmod . \mu):
$$

congruence qui, par hypothèse, n'est point satisfaite.

Quelle que soit la valeur du nombre premier $\alpha$, il est aisé de voir que le nombre premier $\mu$ est de la forme $8 h-3$; donc 2 est racine impaire de $\mu$, et l'on peut dire:

Théorème a. Le nombre 2 est racine primitive de tout nombre premier $\mu$ de la forme $2^{2} \alpha^{2}+1$ ( $\alpha$ étant également un nombre premier), si la congruence

n'est pas satisfaite.

$$
2^{2 \alpha}+1 \equiv 0 \quad(\bmod . \mu)
$$

Si $\alpha$ est différent de $3, \mu$ est de la forme $12 h+5$; par suite le nombre 3 est racine impaire de $\mu$, et on peut dire:

Théorème b. Le nombre 3 est racine primitive de tout nombre premier $\mu$ de la forme $2^{2} \alpha^{2}+1$ ( $\alpha$ étant un nombre premier $>3$ ), si la congruence

n'est pas satisfaite.

$$
3^{2 \alpha}+1 \equiv 0 \quad(\bmod . \mu)
$$

Si $\alpha$ est différent de $5, \mu$ est de la forme $10 h-3$, et par suite 5 est une racine impaire de $\mu$, et l'on peut dire:

Théorème c. Le nombre 5 est racine primitive de tout nombre premier $\mu$ de la forme $2^{2} \alpha^{2}+1$. ( $\alpha$ étant un nombre premier différent de 5), 
12. Oltramare, sur les racines primitives.

si la congruence

n'est pas satisfaite.

$$
5^{2 \alpha}+1 \equiv 0 \quad(\bmod . \mu)
$$

§. 12.

Théorème XII. Si $\mu$ est un nombre premier de la forme $2 \alpha^{m} \beta \cdot \gamma \ldots \varepsilon+1$, tel que la congruence

$$
2^{2 \alpha^{m}}-1 \equiv 0 \quad(\bmod . \mu)
$$

n'est point satisfaile. En posant

$$
\boldsymbol{R}_{k} \equiv \cos \frac{2 k \omega}{\alpha}+\sin \frac{2 k \omega}{\alpha} \sqrt{ }-1 \quad(\bmod . \mu):
$$

$1^{\circ}$. la valeur de l'expression

$$
\sqrt{\alpha^{m-1}}\left(2^{c^{m}} \boldsymbol{R}_{k}\right) \quad(\bmod . \mu)
$$

sera une racine primitive de $\mu$, si ce nombre premier est de la forme $8 h \pm 3$;

$2^{\circ}$. la valeur de l'expression

$$
\frac{-1}{\alpha^{m-1}} \quad(\bmod \mu)
$$

sera une racine primitive de $\mu$, si ce nombre premier est de la forme $8 h \pm 1$.

Nous savons par le Théorème (I.) que:

$1^{\circ}$. si 2 est racine impaire de $\mu$, l'expression $\sqrt{\alpha^{m-1}}\left(2^{\alpha^{m}} R_{k}\right)(\bmod \mu)$ est une racine primitive de ce même nombre premier;

$2^{o}$. si 2 est racine paire de $\mu$, l'expression $\sqrt{ }\left(2^{\alpha^{m}} \boldsymbol{R}_{k}\right)(\bmod . \mu)$ est une racine seconde de ce même nombre premier.

Or comme 2 est racine impaire de tout nombre premier $\mu$ de la forme $\alpha^{m-1}$

$8 h \pm 3$, l'expression $\sqrt{ }\left(2^{\alpha^{m}} \boldsymbol{R}_{k}\right)(\bmod , \mu)$ est une racine primitive de tout nombre premier de cette forme. Comme 2 est racine paire de tout nombre premier de la forme $8 h \pm 1$, l'expression ${ }^{\alpha^{m-1}}\left(2^{\alpha^{m}} R_{k}\right)$ (mod. $\mu$ ) est une racine seconde de tout nombre premier de cette dernière forme.

Si donc on détermine $z$ au moyen de la congruence

$$
\left.\approx \sqrt[\alpha^{m-1}]{2^{\alpha^{m}}} R_{k}+1 \equiv 0 \quad \text { (mod. } \mu\right)
$$

Crelle's Journal f. d. M. Bd. XLIX. Heft 2. 
elle donne

$$
z \equiv \frac{-1}{\sqrt{\alpha^{m-1}}\left(2^{\alpha^{m}} R_{k}\right)} \quad(\bmod . \mu)
$$

comme racine primitive du nombre premier $\mu$.

\$. 13.

En faisant successivement $\alpha=3, \alpha=5, \alpha=7$, on obtient les théorèmes suivants:

Théorème a. Si $\mu$ est un nombre premier de la forme $2.3^{m} \cdot \beta \cdot \gamma \ldots \varepsilon+1(\beta, \gamma, \ldots \varepsilon$ étant des nombres premiers différents entr'eux et plus grands que 3 ), et si la congruence

$$
\text { (1.) } \quad 2^{2.3^{m}}-1 \equiv 0 \quad(\bmod . \mu)
$$

n'est pas satisfaite: en mettant $\mu$ sous la forme $q^{2}+3 p^{2}$ et en désignant par $x_{1}$ et $y_{1}$ une solution particulière de l'équation indéterminée

$$
q x+p y=1 \text { : }
$$

$1^{\circ}$. l'expression

$$
\text { (2.) } \sqrt{ } 3^{m-1}\left(2^{3^{m}-1}\left\{q y_{1}-3 p x_{1}-1\right\}\right) \quad(\bmod . \mu)
$$

représentera une racine primitive du nombre premier $\mu$, si ce nombre est de la forme $8 h \pm 3$;

$2^{\circ}$. l'expression

$$
\frac{-1}{3^{m-1}\left(2^{3^{m}-1}\left\{q y_{1}-3 p x_{1}-1\right\}\right)} \quad(\bmod \mu)
$$

représentera une racine primitive du nombre premier $\mu$, si ce nombre est de la forme $8 h \pm 1$.

Il est facile de voir que la congruence (1.) ne peut être satisfaite si $m=1, m=2, m=3$, auxquels cas les expressions (2.) et (3.) représentent une racine primitive du nombre premier $\mu$.

Théorème b. Si $\mu$ est.un nombre premier de la forme $2.5^{m} \cdot \beta \cdot \gamma \ldots \varepsilon+1(\beta, \gamma, \ldots \varepsilon$ étant également des nombres premiers diffẻrents de 5 et 2 ), et si la congruence

$$
\text { (1.) } \quad 2^{2.5^{m}}-1 \equiv 0 \quad(\bmod , \mu)
$$


19. Oltramare, sur les racines primitives.

n'est pas satisfaite; en mettant $\mu$ sous la forme $q^{2}-5 p^{2}$ et en désignant par $x_{1}$ et $y_{1}$ une solution particulière de l'équation indéterminée

$1^{\circ}$. l'expression

$$
q x+p y=1:
$$

(2.) $\sqrt{ }\left(2^{5^{m-1}-2}\left\{q y_{1}+5 p x_{1}-1+\sqrt{ }\left(-10-2\left(q y_{1}+5 p x_{1}\right)\right)\right\}\right) \quad(\bmod . \mu)$ représentera une racine primitive du nombre premier $\mu$; si ce nombre est de la forme $8 h \pm 3$;

$2^{\circ}$. l'expression

$$
\left.\frac{-1}{5^{m-1}\left(2^{5^{m}-2}\left\{q y_{1}+5 p x_{1}-1+\sqrt{ }\left(-10-2\left(q y_{1}+5 p x_{1}\right)\right)\right\}\right)} \text { (mod. } \mu\right)
$$

représentera une racine primitive du nombre premier $\mu$, si ce nombre est de la forme $8 h \pm 1$.

En supposant successivement $m=1, m=2, m=3$, et en remarquant que, dans ces cas, la congruence (1.) ne peut être satisfaite, on est conduit à admettre que les expressions (2.) ou (3.) représentent des racines primitives de $\mu(\mu=31$ est la seule exception).

Théorème c. Si $\mu$ est un nombre premier de la forme $2.7^{m} \cdot \beta \cdot \gamma \ldots \varepsilon+1(\beta, \gamma, \ldots \varepsilon$ étant également des nombres premiers différents de 7 et 2 ), et si la congruence

n'est pas satisfaite:

$$
\text { (1.) } \quad 2^{2.7^{m}}-1 \equiv 0 \quad(\bmod . \mu)
$$

$1^{\circ}$. l'expression

$$
\text { (2.) } \quad \sqrt{ }\left(2^{7^{m}} R_{k}\right) \quad(\bmod . \mu)
$$

dans laquelle

$$
\boldsymbol{R}_{k} \equiv \frac{1}{2} z \pm \frac{1}{2} \sqrt{ }\left(z^{2}-4\right), \quad z \equiv \frac{1}{3}(v-1), \quad v^{3}-21 v-7 \equiv 0 \quad(\bmod . \mu)
$$

représentera des racines primitives du nombre premier $\mu$, si ce nombre est de la forme $8 h \pm 3$;

$2^{\circ}$. l'expression

$$
\text { (3.) } \frac{-1}{\sqrt{7^{m-1}}\left(2^{7^{m}} R_{k}\right)}(\bmod . \mu)
$$

représentera des racines primitives de $\mu$ si ce nombre est de la forme $8 k \pm 1$. 
En supposant successivement $m=1, m=2, m=3$ et en remarquant que, dans ces cas, la congruence (1.) ne peut être satisfaite, on est conduit à admettre que les expressions (2.) ou (3.) représentent des racines primitives de $\mu(\mu=43$ est la seule exception).

S. 14.

Théorème XIII. Si $\mu$ est un nombre premier de la forme $2^{2} \cdot \alpha^{m} \cdot \beta \cdot \gamma \ldots \varepsilon+1(\alpha, \beta, \gamma, \ldots \varepsilon$ étant également des nombres premiers $)$ tel que la congruence

$$
2^{2^{2} \alpha^{m}}-1 \equiv 0 \quad(\bmod . \mu)
$$

n'est point satisfaite; en posant

$$
\boldsymbol{R}_{k} \equiv \cos \frac{2 k \omega}{\alpha}+\sin \frac{2 k \omega}{\alpha} \sqrt{ }-1 \quad(\bmod . \mu):
$$

la valeur de l'expression

$$
\sqrt{\alpha^{m-1}}\left(2^{\alpha^{m}} \boldsymbol{R}_{k}\right) \quad(\bmod . \mu)
$$

sera une racine primitive de $\mu$.

Ce théorème n'est qu'une conséquence du théorème (I.). En effet le nombre premier $\mu$ est de la forme $8 h-3$ et par suite 2 est une racine impaire de ce nombre et l'expression $\sqrt{ }\left(2^{\alpha^{m}} \boldsymbol{R}_{k}\right)(\bmod , \mu)$ en est une racine primitive.

\$. 15.

En faisant successivement $\alpha=3, \alpha=5, \alpha=7$ on abtient les théorèmes suivants :

Théorème a. Si $\mu$ est un nombre premier de la forme $2^{2} \cdot 3^{m} \cdot \beta \cdot \gamma \ldots \varepsilon+1(\beta, \gamma, \ldots \varepsilon$ étant également des nombres premiers plus grand que 3 ) et si la congruence

$$
\text { (1.) } \quad 2^{2^{2} \cdot 3^{m}}-1 \equiv 0 \quad(\bmod . \mu)
$$

n'est pas satisfaite: en meltant $\mu$ sous la forme $q^{2}+3 p^{2}$ et en désignant par $x_{1}$ et $y_{1}$ une solution particulière de l'équation indélerminée

\section{l'expression}

$$
q x+p y=1:
$$

$$
\text { (2.) } \sqrt{ }\left(2^{3^{m}-1}\left\{4 y_{1}-3 p x_{1}-1\right\}\right) \quad(\bmod . \mu)
$$

représentera une racine primitive du nombre premier $\mu$. 
12. Oltramare, sur les racines primitives.

Il est facile de voir que la congruence (1.) ne peut être satisfaite si $m=1, m=2, m=3$; dans ces cas l'expression (2.) représente une racine primitive de $\mu$.

Théorème b. Si $\mu$ est un nombre premier de la forme $2^{2} \cdot 5^{m} \cdot \beta \cdot \gamma \ldots \varepsilon+1(\beta, \gamma, \ldots \varepsilon$ étant également des nombres premiers différents de 5) et si la congruence

$$
\text { (1.) } \quad 2^{2^{2} \cdot 5^{m}}-1 \equiv 0 \quad(\bmod . \mu)
$$

n'est pas satisfaite: en mettant $\mu$ sous la forme $\varphi^{2}-5 p^{2}$ et en désignant par $x_{1}$ et $y_{1}$ une solution parliculière de l'équation indéterminée

$$
q x+p y=1:
$$

l'expression

$$
\text { (2.) } \sqrt{ }\left(2^{5^{m}-2}\left\{q y_{1}+5 p x_{1}-1+\sqrt{ }\left(-10-2\left(q y_{1}+5 p x_{1}\right)\right)\right\}\right) \quad(\bmod . \mu)
$$

représentera une racine primitive du nombre premier $\mu$.

Il est facile de voir que la congruence (1.) ne peut être satisfaite si $m=1, m=2, m=3$; dans ces cas l'expression (2.) représente une racine primitive de $\mu$.

Théorème c. Si $\mu$ est un nombre premier de la forme $2^{2} \cdot 7^{m} \cdot \beta \cdot \gamma \ldots \varepsilon+1(\beta, \gamma, \ldots \varepsilon$ étant également des nombres premiers différents de 7 ) et si la congruence

$$
\text { (1.) } \quad 2^{2^{2} \cdot 7^{m}}-1 \equiv 0 \quad(\bmod . \mu)
$$

n'est pas satisfaite, l'expression

$$
\text { (2.) } \sqrt{7^{m-1}}\left(2^{7^{m}} R_{k}\right) \quad(\bmod . \mu)
$$

dans laquelle

$$
\boldsymbol{R}_{k} \equiv \frac{1}{2} z \pm \frac{1}{2} \sqrt{ }\left(z^{2}-4\right), \quad z \equiv \frac{1}{3}(v-1), \quad v^{3}-21 v-7 \equiv 0 \quad(\bmod , \mu)
$$

représentera une racine primitive du nombre premier $\mu$.

Il est facile de voir que la congruence (1.) ne peut être satisfaite si $m=1, m=2, m=3$; dans ces cas l'expresṣion (2.) représente une racine primitive de $\mu$.

S. 16.

On peut déduire du théoréme (I.) la proposition suivante:

Théorème XIV. Si $\mu$ est un nombre premier de la forme $2^{s} \alpha^{m}+1$ ( $\alpha$ étant également un nombre premier) la valeur de l'expression 


$$
\sqrt{c^{m-1}}\left[\left(\boldsymbol{r}_{t}\right)^{\alpha^{m}}\left(\cos \frac{2 k \omega}{\alpha}+\sin \frac{2 k \omega}{\alpha} \sqrt{ }-1\right)\right] \quad(\bmod . \mu)
$$

est une racine primitive du nombre premier $\mu$, si $r_{t}$ est une racine impaire de ce nombre.

En supposant $s=1$ et $m$ plus grand que $1 ; 2$ est racine impaire de $\mu$ qui est alors de la forme $8 h+3$ et l'on peut établir la proposition suivante :

Théorème $X V$. Si $\mu$ est un nombre premier de la forme $2 \alpha^{m}+1$ ( $\alpha$ étant un nombre premier et $m>1$ ), la valeur de l'expression

$$
\sqrt{\alpha^{m-1}}\left(2^{\alpha^{m}}\left\{\cos \frac{2 k \omega}{\alpha}+\sin \frac{2 k \omega}{\alpha} \sqrt{ }-1\right\}\right)(\bmod . \mu) .
$$

En admettant que $\alpha$ soit successivement égal à $3,5,7$ on obtient les théorèmes particuliers suivants:

Théorème a. Si $\mu$ est un nombre premier de la forme $2.3^{m}+1$, ce nombre pourra également se mettre sous la forme $q^{2}+3 p^{2}$, et en désignant par $x_{1}$ et $y_{1}$ une solution particulière de l'équation indéterminée la valeur de l'expression

$$
q \dot{x}+p y=1:
$$

$$
\sqrt{ } 3^{m-1}\left(2^{3^{m}-1}\left(q y_{1}-3 p x_{1}-1\right)\right) \quad(\bmod . \mu)
$$

sera racine primitive du nombre premier $\mu$.

Théorème b. Si $\mu$ est un nombre premier de la forme $2.5^{m}+1$, ce nombre premier pourra également se mettre sous la forme $q^{2}-5 p^{2}$, et en désignant par $x_{1}$ et $y_{1}$ une solution particulière de l'équation indéterminée

la valeur de l'expression

$$
q x+p y=1:
$$

$$
\sqrt{5^{m-1}}\left(2^{5^{m}-2}\left\{q y_{1}+5 p x_{1}-1+\sqrt{ }\left(-10-2\left(q y_{1}+5 p x_{1}\right)\right)\right\}\right) \quad(\bmod . \mu)
$$

sera une racine primitive du nombre premier $\mu$.

Théorème c. Si $\mu$ est un nombre premier de la forme $2.7^{m}+1$, l'expression

$$
\sqrt{7^{m-2}}\left(2^{7^{m}} R_{k}\right) \quad(\bmod \mu)
$$


dans laquelle

$$
\boldsymbol{R}_{k} \equiv \frac{1}{2}+\frac{1}{2} \sqrt{ }\left(z^{2}-4\right), \quad z \equiv \frac{1}{3}(v-1), \quad v^{3}-21 v-7 \equiv 0 \quad(\bmod . \mu)
$$

représentera une racine primitive du nombre premier $\mu$.

\section{S. 17.}

On déduit aisément du théorème (II.) la proposition suivante:

Théorème XVI. Si $\mu$ est un nombre premier de la forme $2^{s} .3^{m}+1$, la valeur de l'expression

$$
r^{3} \sqrt{3-1}\left(\frac{1}{2}\left(q y_{1}-3 p x_{1}-1\right)\right)
$$

sera une racine primitive de $\mu$, si $r$ est une racine impaire de ce nombre et si, en mettant $\mu$ sous la forme $q^{2}+3 p^{2}, x_{1}$ et $y_{1}$ représentent une solution particulière de l'équation indéterminée

$$
q x+p y=1 \text {. }
$$

\$. 18.

Si $\mu$ est un nombre premier de la forme $2^{s} \alpha^{m}+1(s>1)$, dans laquelle $\alpha$ est un nombre premier différent de 3 , il est facile de reconnaitre que ce nombre premier est de la forme $12 h+5$. En effet, en supposant $\alpha$ de la forme $3 t+1$, on a $\mu=12 T+2^{s}+1$; il en résulte que $s$ doit être de la forme 2p; car s'il en était autrement, le nombre premier $\mu$ serait divisible par 3, ce qu'on ne peut admettre. Si donc nous posons

$$
2^{s} \alpha^{m}+1=12 \ell+5=12 T+2^{2 p}+1
$$

il en résultera

$$
h=T+\frac{2^{2 p-2}-1}{3}:
$$

égalité qui donne pour $h$ un nombre entier.

En supposant $\alpha$ de la forme $3 t+2$, on a $\mu=12 T+2^{s+m}+1$; il en résulte que $s+m$ est de la forme $2 p$, car autrement $\mu$ serait divisible par 3 . $\mathrm{Si}$ donc nous posons

il en résulterá

$$
2^{s} \alpha^{m}+1=12 h+5=12 T+2^{2 p}+1,
$$

$$
h=T+\frac{2^{2 p-2}-1}{3}:
$$

égalité qui donne pour $h$ un nombre entier.

Il résulte de cette observation que 3 est racine impaire de tout nombre premier de la forme $2^{s} \alpha^{m}+1$, et par suite le théorème XIV nous permet d'établir la proposition suivante: 
Théorème XVII. Si $\mu$ est un nombre premier de la forme $2^{s} \alpha^{m}+1$ $(s>1$ et $\alpha$ un nombre premier différent de 3), la valeur de l'expression

$$
\sqrt{\alpha^{m-1}}\left(3^{\alpha^{m}}\left\{\cos \frac{2 k \omega}{\alpha}+\sin \frac{2 k \omega}{\alpha} \sqrt{ }-1\right\}\right) \quad(\bmod . \mu)
$$

sera une racine primitive du nombre premier $\mu$.

En faisant successivement $\alpha=5, \alpha=7$ on obtient:

Théorème a. Si $\mu$ est un nombre premier de la forme $2^{s} .5^{m}+1$ $(s>1)$, ce nombre pourra égalenent être mis sous la forme $q^{2}-5 p^{2}$, et en désignant par $x_{1}$ et $y_{1}$ une solution particulière de l'équation indélerminée

l'expression

$$
q x+p y=1:
$$

$$
\sqrt{s^{m-1}}\left(\frac{3^{s m}}{4}\left\{q y_{1}+5 p x_{1}-1+\sqrt{ }\left(-10-2\left(q y_{1}+5 p x_{1}\right)\right)\right\}\right) \quad(\bmod . \mu)
$$

sera une racine primitive du nombre premier $\mu$.

Théorème b. Si $\mu$ est un nombre premier de la forme $2^{s} .7^{m}+1$ $(s>1)$, l'expression

dans laquelle

$$
\sqrt{7^{m-1}}\left(3^{7^{m}} \boldsymbol{R}_{k}\right) \quad(\bmod . \mu)
$$

$$
\left.\boldsymbol{R}_{k} \equiv \frac{1}{2} z \pm \frac{1}{2} \sqrt{ }\left(z^{2}-4\right), \quad z \equiv \frac{1}{3}(v-1), \quad v^{3}-21 v-7 \equiv 0 \quad \text { (mod. } \mu\right),
$$

représentera une racine primitive du nombre premier $\mu$.

ऽ. 19.

On peut encore établir comme cas particulier du théorème (I.) la proposition suivante:

Théorème XVIII. Si $\mu$ est un nombre premier de la forme $2^{s} \cdot \alpha^{m} \cdot \beta \cdot \gamma \ldots \varepsilon+1(\alpha, \beta, \gamma, \ldots \varepsilon$ étant des nombres premiers) tel que la congruence

$$
r^{2^{s} \alpha^{m}}-1 \equiv 0 \quad(\bmod . \mu)
$$

(dans laquelle $r$ est une racine impaire de $\mu$ ) n'est pas satisfaite; en posant

$$
\boldsymbol{R}_{k} \equiv \cos \frac{2 k \omega}{\alpha}+\sin \frac{2 k \omega}{\alpha} \sqrt{ }-1 \quad(\bmod . \mu):
$$

la valeur de l'expression

$$
\boldsymbol{\alpha}^{\boldsymbol{\alpha}-1}\left(\boldsymbol{r}^{\boldsymbol{\alpha}^{m}} \boldsymbol{R}_{k}\right) \cdot(\bmod , \mu)
$$

sera une racine primitive de $\mu$. 
Voici quelques cas particuliers de ce théorème.

Théorème a. Si le nombre premier $\mu=10 n \pm 3$ est de la forme $2^{s} \cdot 3^{m} \cdot \beta \cdot \gamma \ldots \varepsilon+1$, ce nombre pourra également se mettre sous la forme $y^{2}+3 p^{2}$, et en désignant par $x_{1}$ et $y_{1}$ une solution particulière de l'équation indéterminée

la valeur de l'expression

$$
q x+p y=1:
$$

$$
\sqrt{\left(\frac{3^{m-1}}{2}\left(q y_{1}-3 p x_{1}-1\right)\right)}(\bmod . \mu)
$$

sera une racine primitive de $\mu$, si la congruence

$$
5^{2^{s} \cdot 3^{m}}-1 \equiv 0 \quad(\bmod . \mu)
$$

n'est pas satisfaite.

Soient $m=1$ et $s=3$.

Théorème $a^{\prime}$. Si le nombre premier $\mu=10 n \pm 3$ (excepté 313) est de la forme $2^{3} .3 . \beta . \gamma \ldots \varepsilon+1$; ce nombre pourra également se mettre sous la forme $q^{2}+3 p^{2}$, et en désignant par $x_{1}$ et $y_{1}$ une solution particulière de l'équation indéterminée:

la valeur de l'expression

$$
q x+p y=1
$$

$$
\frac{5^{3}}{2}\left(q y_{1}-3 p x_{1}-1\right) \quad(\bmod . \mu)
$$

sera une racine primitive de $\mu$.

Soient $m=1$ et $s=4$.

Théorème $a^{\prime \prime}$. Si le nombre premier $\mu=10 n \pm 3$ est. de la forme $2^{4} .3 . \beta . \gamma \ldots \varepsilon+1$; ce nombre pourra également se mettre sous la forme $q^{2}+p^{2}$, et en désignant par $x_{1}$ et $y_{1}$ une solution particulière de l'équation indéterminée

la valeur de l'expression

$$
q x+p y=1:
$$

$$
\frac{5^{3}}{2}\left(q y_{1}-3 p x_{1}-1\right) \quad(\bmod \mu)
$$

sera une racine primitive de $\mu$.

Soient $m=2$ et $s=3$.

Théorème b. Si le nombre premier $\mu=10 n \pm 3$ est de la forme. $2^{3} \cdot 3^{2} \cdot \beta \cdot \gamma \ldots \varepsilon+1$, ce nombre premier pourra également se mettre sous Crelle's Journal f. d. M. Bd. XLIX. Heft 2. 
la forme $q^{2}+3 p^{2}$, et en désignant par $x_{1}$ et $y_{1}$ une solution particuliere de l'équation indéterminée

la valeur de l'expression

$$
q x+p y=1:
$$

$$
\sqrt[3]{\left(\frac{5^{9}}{2}\left(q y_{1}-3 p x_{1}-1\right)\right)(\bmod . \mu)}
$$

sera une racine primitive de $\mu$, si la congruence

n'est pas satisfaite.

$$
5^{72}-1 \equiv 0 \quad(\bmod . \mu)
$$

Théorème c. Si $\mu$ est un nombre premier de la forme $2^{3} \cdot 5 \cdot \beta \cdot \gamma \ldots \varepsilon+1(\beta, \gamma, \ldots \varepsilon$ étant également dẻs nombres premiers plus grands que 5), ce nombre pourra également se mettre sous la forme $q^{2}-5 p^{2}$, et en désignant par $x_{1}$ et $y_{1}$ une solution particulière de l'équation indéterminée

l'expression

$$
q x+p y=1:
$$

$$
\frac{3^{5}}{4}\left\{q y_{1}+5 p x_{1}-1+\sqrt{ }\left(-10-2\left(q y_{1}+5 p x_{1}\right)\right)\right\} \quad(\bmod . \mu)
$$

sera une racine primitive du nombre premier $\mu$.

Théorème $c^{\prime}$. Si $\mu$ est un nombre premier de la forme $2^{4} .5 \cdot \beta \cdot \gamma \ldots \varepsilon+1(\beta, \gamma, \ldots \varepsilon$ étant des nombres premiers plus grands que 5), ce nombre pourra également se metlre sous la forme $q^{2}-5 p^{2}$, et en désignant par $x_{1}$ et $y_{1}$ une solution particulière de l'équation indéterminée

l'expression

$$
q x+p y=1:
$$

$$
\frac{3^{5}}{4}\left\{4 y_{1}+5 p x_{1}-1+\sqrt{ }\left(-10-2\left(q y_{1}+5 p x_{1}\right)\right)\right\} \quad(\bmod . \mu)
$$

sera une racine primitive du nombre $\mu$, si la congruence

n'est pas satisfaite.

$$
3^{40}+1 \equiv 0 \quad(\bmod . \mu)
$$

Théorème $c^{\prime \prime}$. Si $\mu$ est un nombre premier de la forme $2^{5} .5 . \beta . \gamma, \ldots \varepsilon+1(\beta, \gamma, \ldots \varepsilon$ étant des nombres premiers plus grands que 5), ce nombre pourra également se mettre sous la forme $q^{2}-5 p$, et en désignant par $x_{1}$ et $y_{1}$ une solution particulière de l'équation indélerminée

$$
q x+p y=1:
$$


l'expression

$$
\frac{3^{5}}{4}\left\{q y_{1}+5 p x_{1}-1+\sqrt{ }\left(-10-2\left(q y_{1}+5 p x_{1}\right)\right)\right\} \quad(\bmod . \mu)
$$

sera une racine primitive du nombre premier $\mu$, si la congruence

n'est pas satisfaite.

$$
\left(3^{80}+1\right)\left(3^{40}+1\right) \equiv 0 \quad(\bmod . \mu)
$$

Théorème d. Si $\mu$ est un nombre premier de la forme $2^{3} \cdot 7 . \beta . \gamma \ldots \varepsilon+1(\beta, \gamma, \ldots \varepsilon$ étant des nombres premiers différents de 3$)$, l'expression

dans laquelle

$$
\mathbf{3}^{7} \boldsymbol{R}_{k}(\bmod . \mu),
$$

$$
\boldsymbol{R}_{k} \equiv \frac{1}{2} z \pm \frac{1}{2} \sqrt{ }\left(z^{2}-4\right), \quad z \equiv \frac{1}{3}(v-1), \quad v^{3}-21 v-7 \equiv 0 \quad(\bmod . \mu),
$$

représentera une racine primitive de $\mu$.

Théorème $d^{\prime}$. Si le nombre premier $\mu=28 n \pm 5$ ou $28 n \pm 11$ ou $28 n \pm 13$ est de la forme $2^{s} \cdot 3^{m} \cdot \beta \cdot \gamma \ldots \varepsilon+1$, ce nombre premier pourra également se mettre sous la forme $q^{2}+3 p^{2}$, et en désignant par $x_{1}$ et $y_{1}$ une solution particulière de l'équation indéterminée

la valeur de l'expression

$$
q x+p y=1:
$$

$$
\sqrt{\left(\frac{7^{3^{m}}}{2}\left(q y_{1}-3 p x_{1}-1\right)\right) \quad(\bmod . \mu)}
$$

sera une racine primitive de $\mu$, si la congruence

n'est pas satisfaite.

$$
7^{2^{s} \cdot 3^{m}}-1 \equiv 0 \quad(\bmod . \mu)
$$

Soient $m=1$ et $s=3$.

Théor ème $d^{\prime \prime}$. Si le nombre premier $\mu=28 n \pm 5$ ou $28 n \pm 11$ ou $28 n \pm 13$ est de la forme $2^{3} .3 . \beta . \gamma \ldots \varepsilon+1$, ce nombre premier pourra également se mettre sous la forme $q^{2}+3 p^{2}$ et en désignant par $x_{1}$ et $y_{1}$ une solution particulière de l'équation indéterminée

la valeur de l'expression

$$
q x+p y=1
$$

$$
\frac{7^{3}}{2}\left(q y_{1}-3 p x_{1}-1\right) \quad(\bmod . \mu)
$$

sera une racine primitive de $\mu_{t}$ 
Théorème $d^{\prime \prime \prime}$. Si le nombre premier $\mu=44 n \pm 3$ ou $44 n \pm 13$ ou $44 n \pm 15$ ou $44 n \pm 17$ ou $44 n \pm 21$ est de la forme $2^{3} .3 . \beta . \gamma \ldots \varepsilon+1$ ce nombre pourra également se meltre sous la forme $q^{2}+3 p^{2}$, et en designant par $x_{1}$ ef $y_{1}$ une solution particulière de l'équation indéterminée

la valeur de l'expression

$$
q x+p y=1:
$$

$$
\frac{11^{3}}{2}\left(q y_{1}-3 p x_{1}-1\right) \quad(\bmod . \mu)
$$

sera une racine primitive de $\mu$.

Genève 23 Février 1854. 